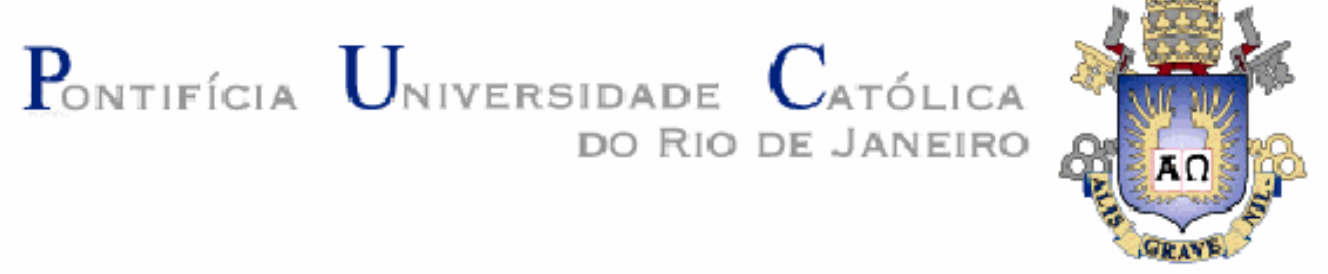

Tayana Aparecida Rigueira

Capital Requerido via Simulação Estocástica aplicado ao Seguro de Vida e Fundo de Pensão

Dissertação de Mestrado

Dissertação apresentada ao Programa de Pósgraduação em Ciências Atuariais da PUC-Rio como requisito parcial para obtenção do título de Mestre em Ciências Atuariais.

Orientadora: Fernanda Chaves Pereira

Rio de Janeiro

Março de 2009 


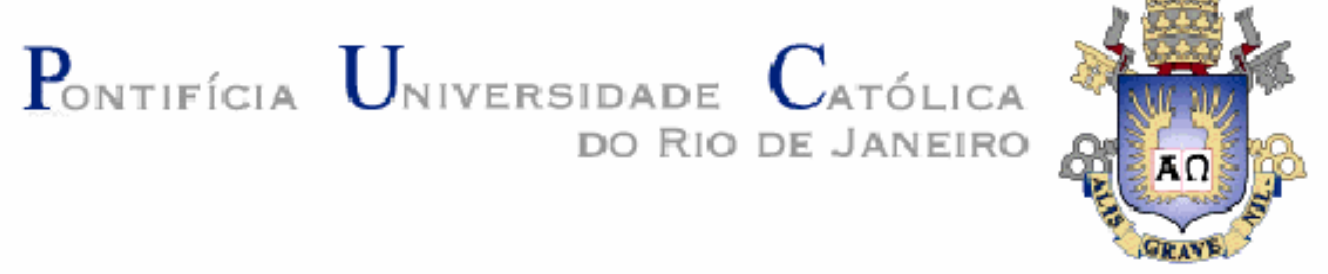

Tayana Aparecida Rigueira

\section{Capital Requerido via Simulação Estocástica aplicado ao Seguro de Vida e Fundo de Pensão}

Dissertação apresentada como requisito parcial para obtenção do grau de Mestre pelo Programa de Pósgraduação em Ciências Atuariais da PUC-Rio. Aprovada pela Comissão Examinadora abaixo assinada.

Prof ${ }^{a}$. Fernanda Chaves Pereira

Orientadora

Instituto de Gestão de Riscos Financeiros e Atuariais da PUC-Rio

Prof. Álvaro de Lima Veiga Filho

Departamento de Engenharia Elétrica - PUC-Rio

Prof. Ricardo Milton Frischtak Departamento de Métodos Estatísticos da UFRJ

Prof. Nizar Messari

Coordenador Setorial do Centro de Ciências Sociais - PUC-Rio

Rio de Janeiro, 30 de março de 2009 
Todos os direitos reservados. É proibida a reprodução total ou parcial do trabalho sem autorização da universidade, da autora e do orientador.

\section{Tayana Aparecida Rigueira}

Graduou-se em Ciências Atuariais na UFRJ (Universidade Federal do Rio de Janeiro) e Estatística na ENCE (Escola Nacional de Ciências Estatísticas) em 2006

Ficha Catalográfica

Rigueira, Tayana Aparecida

Capital requerido via simulação estocástica aplicado ao seguro de vida e fundo de pensão / Tayana Aparecida Rigueira ; orientador: Fernanda Chaves Pereira. -2009 .

107 f. : il.(color.) ; $30 \mathrm{~cm}$

Dissertação (Mestrado em Gestão de Riscos Financeiros e Atuariais)-Pontifícia Universidade Católica do Rio de Janeiro, Rio de Janeiro, 2009.

Inclui bibliografia

1. Gestão de riscos financeiros e atuariais Teses. 2. Atuária. 3. Capital requerido para solvência. 4. Modelo interno. 5. Solvência. 6. Simulação de Monte Carlo. 7. Múltiplos decrementos. 8. Seguro de vida. 9. Fundo de pensão. I. Pereira, Fernanda Chaves. II. Pontifícia Universidade Católica do Rio de Janeiro. Instituto de Gestão de Riscos Financeiros e Atuariais. III. Título. 
Dedico aos meus pais, minha irmã e minha avó (in memoriam). 


\section{Agradecimentos}

A Deus, por acreditar que a nossa existência pressupõe outra infinitamente superior.

Aos meus pais, Vera Novo e Heitor Rigueira, pela educação, atenção, pelas conversas, conselhos, amor e compreensão a todo momento. Pelas orações de minha mãe sempre me deixando tranqüila e pela ajuda de meu pai que é meu verdadeiro mestre.

A minha irmã, Mayara Rigueira, por me apoiar nesta nova etapa.

Aos meus "antigos" amigos, por compreenderem minha ausência e por serem sempre tão companheiros e leais.

Aos meus novos amigos Mariana, Luciene, Bruna e Alexandre pelo apoio ao longo desses 2 anos, pela amizade e por estarem sempre dispostos a me ouvir.

Ao IAPUC e FUNENSEG, pelos auxílios concedidos e estrutura oferecida.

Agradeço a minha orientadora Professora Fernanda Chaves Pereira pelos

estímulos e, por percorrer novos caminhos, ouvir todas as questões, dúvidas e problemas que surgiam durante o processo.

Aos professores que participaram da Comissão examinadora pelas críticas construtivas ao trabalho.

A todos os professores e funcionários do Departamento pelos ensinamentos e pela ajuda. 


\section{Resumo}

Rigueira, Tayana Aparecida; Pereira, Fernanda Chaves. Capital Requerido via Simulação Estocástica aplicado ao Seguro de Vida e Fundo de Pensão. Rio de Janeiro, 2009. 107p. Dissertação de Mestrado - Instituto de Gestão de Riscos Financeiros e Atuariais, Pontifícia Universidade Católica do Rio de Janeiro.

As grandes mudanças mundiais ocorridas a partir da década de 70 tornaram o mercado financeiro mais volátil, exigindo medidas que minimizassem o risco do sistema. Iniciou-se assim a utilização de métodos baseados em análise de risco. Este novo conceito tem como princípio a mensuração e a garantia da solvência de uma empresa para que possa operar resguardando-se dos riscos econômicos a que esteja sujeita, com um alto nível de confiança, dado um horizonte de tempo pré definido, utilizando para isso modelos internos de gestão. $\mathrm{O}$ modelo proposto nesta dissertação para o risco de subscrição se baseia na utilização de tábuas de múltiplos decrementos e Simulação de Monte Carlo. Foram aplicadas técnicas de Solvency Capital Requirement (SCR) e Minimum Capital Requirement (MCR), bem como avaliadas suas relações com as provisões técnicas em aplicações de seguro de vida e fundos de pensão, nesta situação considerados dois casos: só tendo participantes ativos no grupo e outro incluindo-se participantes assistidos.

\section{Palavras-Chave}

Capital requerido para solvência, modelo interno, solvência, simulação de Monte Carlo, múltiplos decrementos, seguro de vida, fundo de pensão. 


\section{Abstract}

Rigueira, Tayana Aparecida; Pereira, Fernanda Chaves. Capital Requirement by Stochastic Simulation applied to Life Insurance and Pension Fund. Rio de Janeiro, 2009. 107p. MSc. Dissertation - Instituto de Gestão de Riscos Financeiros e Atuariais, Pontifícia Universidade Católica do Rio de Janeiro.

The major global changes that occurred from the 70's became financial market more volatile, requiring measures that minimize the risk of the system. It started the use of methods based on risk analysis. This new concept has in principle the measurement and ensuring the solvency of a company that can operate safeguarding themselves from the economic risks, with a high level of confidence, given a pre defined time, using it for internal models management. The proposed model in this dissertation to the risk of subscription is based on the use of tables of multiple decrements and Monte Carlo Simulation. Techniques were applied to Solvency Capital Requirement (SCR) and Minimum Capital Requirement (MCR) and evaluated its relationship with the technical provisions in life insurance applications and pension funds, in this last two cases were considered: only active participants in the group, and another one including other participants attended.

\section{Keywords}

Solvency capital requirement, internal model, solvency, Monte Carlo Simulation, multiple decrements, life insurance, pension fund. 


\section{Sumário}

$\begin{array}{ll}\text { 1. Introdução } & 15\end{array}$

2. Conceitos de Capital 19

2.1. Tipos de Riscos 20

2.2. Medidas de Risco 24

3. Mensuração de Capital 28

3.1. Relação entre SCR, MCR e Provisões Técnicas 28

3.2. Solvency Capital Requeriment $\quad 30$

3.2.1. Fórmula Padrão 31

3.2.2. Modelos Internos $\quad 32$

3.3. Minimum Capital Requirement $\quad 34$

4. Metodologia 36

4.1. Tábuas de Mortalidade e Invalidez $\quad 38$

4.1.1. Uma Variável Aleatória $\quad 38$

4.1.2. Duas Variáveis Aleatórias $\quad 39$

4.1.3. Associando a Tábua de Único Decremento 42

4.1.4. Construção da Tábua de Múltiplos Decrementos 46

4.2. Simulação 48

4.2.1. Simulação de Monte Carlo $\quad 50$

5. Análise dos Resultados - Seguro de Vida 53

5.1. Base de Dados $\quad 54$

5.2. Modelagem $\quad 55$

5.3. Resultados $\quad 56$

5.3.1. Necessidade de Capital 56

$\begin{array}{ll}\text { 5.3.2. Análise dos Modelos } & 65\end{array}$

5.3.3. Cálculo Resolução versus Modelo Interno $\quad 68$

6. Análise dos Resultados - Fundo de Pensão 71

6.1. Base de Dados 72

$\begin{array}{ll}\text { 6.2. Modelagem } & 74\end{array}$

$\begin{array}{ll}\text { 6.3. Resultados } & 75\end{array}$

6.3.1. Necessidade de Capital $\quad 75$ 
7. Conclusão

8. Bibliografia

9. Anexo 1

10. Anexo 2

106

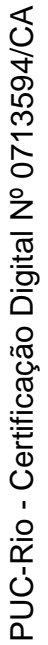




\section{Lista de figuras}

Figura 3.1: Definição do valor para as provisões técnicas e para o SCR das entidades

Figura 5.1: Distribuição do ganho líquido do modelo base (juros de

$4 \%$ a.a.) - em milhões

Figura 5.2: Distribuição do sinistro do modelo base (juros de $4 \%$ a.a.)

- em milhões

Figura 5.3: Distribuição do ganho líquido variando os juros (AT 83)

- em milhões

Figura 5.4: Distribuição do ganho líquido variando tábuas biométricas (juros de 4\% a.a.) - em milhões

Figura 5.5: Distribuição do ganho líquido variando taxa de rotatividade (juros de 4\% a.a.) - em milhões

Figura 5.6: Percentual do SCR em relação ao ganho líquido variando as tábuas biométricas

Figura 5.7: Percentual do SCR em relação ao ganho líquido variando a taxa de rotatividade

Figura 5.8: Valores do SCR variando a medida de risco - em milhões

Figura 6.1: Distribuição do ganho líquido do modelo base (juros de $4 \%$ a.a.) - caso 1 (em milhões)

Figura 6.2: Distribuição do ganho líquido do modelo base (juros de $4 \%$ a.a.) - caso 2 (em milhões)

Figura 6.3: Distribuição do ganho líquido variando os juros no caso 1

(GAM 83) - em milhões

Figura 6.4: Distribuição do ganho líquido variando os juros no caso 2

(GAM 83) - em milhões

Figura 6.5: Distribuição do ganho líquido variando tábuas biométricas no caso 1 (juros de $4 \%$ a.a.) - em milhões

Figura 6.6: Distribuição do ganho líquido variando tábuas biométricas no caso 2 (juros de $4 \%$ a.a.) - em milhões

Figura 6.7: Distribuição do ganho líquido variando taxa de rotatividade no caso 1 (juros de $4 \%$ a.a.) - em milhões

Figura 6.8: Distribuição do ganho líquido variando taxa de rotatividade no caso 2 (juros de $4 \%$ a.a.) - em milhões

Figura 6.9: Percentual do SCR em relação à reserva variando as tábuas biométricas caso 1

Figura 6.10: Percentual do SCR em relação a reserva variando as tábuas biométricas caso 2

Figura 6.11: Percentual do SCR em relação a reserva variando as rotatividades caso 1 
Figura 6.12: Percentual do SCR em relação a reserva variando as rotatividades caso 2

Figura 6.13: Valores de SCR variando a medida de risco (caso 1) - em milhões

Figura 6.14: Valores de SCR variando a medida de risco (caso 2) - em milhões 


\section{Lista de tabelas}

Tabela 5.1: Hipóteses utilizadas nos modelos 56

Tabela 5.2: Valor esperado do ganho líquido do modelo base 56

Tabela 5.3: Valores do ganho médio, margem de risco e SCR (AT 83)

- em milhões

Tabela 5.4: Valores do ganho médio, margem de risco e SCR (CSO 80

e AT 2000) - em milhões

60

Tabela 5.5: Valores do ganho médio, margem de risco e SCR (rotatividade de $15 \%$ a.a. e 30\% a.a.) - em milhões 61

Tabela 5.6: Valor do MCR variando as tábuas biométricas - em milhões $\quad 63$

Tabela 5.7: Valor do MCR variando as taxas de rotatividade - em milhões $\quad 64$

Tabela 5.8: Valores esperados para 1 ano do modelo base - em milhões $\quad 65$

Tabela 5.9: Valores esperados do modelo com tábua CSO 80 - em milhões 66

Tabela 5.10: Valores esperados do modelo com tábua AT 2000

- em milhões 66

Tabela 5.11: Taxa de Sinistralidade para as três tábuas utilizadas $\quad 67$

Tabela 5.12: Valores esperados do modelo com taxa de rotatividade de $15 \%$ a.a. - em milhões

Tabela 5.13: Valores esperados do modelo com taxa de rotatividade de $30 \%$ a.a. - em milhões

68

Tabela 5.14: Taxa de Sinistralidade para as três taxas de rotatividade utilizadas

Tabela 5.15: Capital mínimo requerido segundo forma de cálculo variando a taxa de juros

Tabela 6.1: Hipóteses utilizadas nos modelos

Tabela 6.2: Reserva Matemática do modelo base nos casos 1 e 2 (juros de $4 \%$ a.a.)

Tabela 6.3: Valores do ganho médio, margem de risco e SCR nos casos 1 e 2 (GAM 83) - em milhões

Tabela 6.4: Valores do ganho médio, margem de risco e SCR nos casos 1 e 2 (AT 83) - em milhões

Tabela 6.5: Valores do ganho médio, margem de risco e SCR nos casos 1 e 2 (AT 2000) - em milhões

Tabela 6.6: Valores do ganho médio, margem de risco e SCR nos casos 1 e 2 (rotatividade de $8 \%$ a.a.) - em milhões

Tabela 6.7: Valores do ganho médio, margem de risco e SCR nos casos 1 e 2 (rotatividade de $15 \%$ a.a.) - em milhões

Tabela 6.8: Valor do MCR variando as tábuas biométricas nos casos 1 e 2 - em milhões 
Tabela 6.9: Valor do MCR variando as taxas de rotatividade nos casos 1

e 2 -em milhões

Tabela 6.10: Valor presente dos valores esperados do modelo base

- em milhões

Tabela 6.11: Valor presente dos valores esperados do modelo com tábua AT 83 - em milhões

Tabela 6.12: Valor presente dos valores esperados do modelo com tábua AT 2000 - em milhões

Tabela 6.13: Valor presente do valor esperado do benefício para as três tábuas utilizadas - em milhões

Tabela 6.14: Valor presente dos valores esperados do modelo com taxa de rotatividade de $8 \%$ a.a. - em milhões

Tabela 6.15: Valor presente dos valores esperados do modelo com taxa de rotatividade de $15 \%$ a.a. - em milhões

Tabela 6.16: Valor presente dos valores esperados do modelo base

- em milhões

Tabela 6.17: Valor presente dos valores esperados do modelo com tábua AT 83 - em milhões

Tabela 6.18: Valor presente dos valores esperados do modelo com tábua AT 2000 - em milhões

Tabela 6.19: Valor presente dos valores esperados do benefício para as três tábuas utilizadas - em milhões

Tabela 6.20: Valor presente dos valores esperados do modelo com taxa de rotatividade de $8 \%$ a.a. - em milhões

Tabela 6.21: Valor presente dos valores esperados do modelo com taxa de rotatividade de $15 \%$ a.a. - em milhões 


\section{Lista de quadros}

Quadro 6.1: Freqüência de indivíduos em cada faixa etária

Quadro 6.2: Freqüência de indivíduos em cada faixa de tempo de empresa nos dois casos

Quadro 6.3: Freqüência de indivíduos em relação ao salário anual nos dois casos

Quadro 6.4: Percentual do benefício recebido pela idade de entrada em aposentadoria nos dois casos 